\title{
COLOR AND SEX IN THE INDIAN WALKING STICK, DIXIPPUS MOROSUS
}

\author{
By A. J. Mangelsdorf,
}

Harvard University, Bussey Institution.

In cultures of the Indian walking-stick, Dixippus morosus, reared in the insectary at the Bussey Institution, it was observed that in each generation there was a wide range of coloration varying from a uniform green in some individuals to a brownish black in others. Since this insect usually reproduces parthenogenetically the question as to the behavior in inheritance of the several color types appeared to be one of considerable interest.

Dobkiewicz (1912) had already shown that the color of these insects is influenced by their surroundings. He reared them in cages lined with colored paper, and found that those in green and yellow cages remained light green throughout their lives, while those in red and black cages had become quite black by the time they had reached sexual maturity. However, the occurrence in our cultures under what appeared to be a uniform environment of green and dark brown individuals, in addition to a number which were intermediate between the two extremes, suggested that there might be inherent individual differences in reactivity to a background of a given color.

To obtain further information on this question two females, one a light green and the other a dark brown, were isolated and their eggs saved. The eggs are dropped at the rate of two or three a day, and egg-laying may extend over a period of three to five months. Over one hundred eggs were obtained from each female. They were placed in wide-mouthed bottles stoppered with a cotton plug, and were stored in a damp situation in the insectary. In about five months after the first eggs were laid, hatching began.

Four cages were constructed,-two were lined with light yellowish-green cheesecloth and two with the same material of a very dark red color. After a number of eggs from both the green 
and the brown parent had hatched, twenty-four young from each female were selected and divided into two lots of twelve each. One lot was then placed in a green cage, the other in a red cage.

The insects at this stage were uniformly of a dull green color. They were fed each night with shoots of Tradescantia fluminensis, the remains of which were removed each morning to avoid obscuring the background.

No change could be observed after either the first or the second moult. After the third moult, however, those in the green cages had taken on a clearer, lighter shade of green, while those in the red cages had become perceptibly darker. After the fourth moult the difference was striking,- - those in the green cages remained a clear green, while those in the red cages were dark brown. There was but little difference in the rate of change between the progenies of the two females. Those from the green parent showed, if anything, a more rapid change to brown than did those from the brown parent.

To determine whether the change to dark brown could be effected in the later stages of the life history, two individuals were transferred from each of the green cages and placed with their sisters in a red cage After passing through several moults, they became almost dark as those which had occupied the red cages from the beginning of the experiment

At the same time two brown individuals were removed from each of the red cages and were placed with their sisters in the green cages to determine whether the color change to brown is reversible. In each case the middle pairs of legs of the transferred individuals were removed to distinguish them from the others. After several moults they had taken on a somewhat lighter shade, and when mature they were of an indefinite greyish color; but the dark pigment was never completely resorbed. The middle legs, however, which were regenerated under the influence of the green background were of a peculiar bluish-green shade, their color presenting a striking contrast to that of the rest of the body.

In each of these cases no differences could be observed in the reactivity of the two progenies. 
After the insects had attained sexual maturity, two other green individuals were transferred to red cages and two dark brown individuals to green cages. No color change was observed. Apparently the color present after the last moult is permanent.

The question as to the reasons for the original variations in color of individuals occupying the same environment as found in our cultures remains unanswered. The only explanation which suggests itself is the following: The insects feed during the early part of the night attaching themselves before dawn to a branch or any other suitable support, and remaining motionless in the same spot throughout the day. They show a marked tendency, in captivity at any rate, to return to the same support day after day. If the slight differences which exist in the illumination and background are sufficient to bring about the differences in color, this habit may be responsible for the observed variation.

Fryer (1913), working with a bisexual walking-stick, Clitumnus cuniculus, from Ceylon, in which both yellow and green forms are found, interpreted the color differences as being inherited. The color, according to his hypothesis, is due to action of a single factor pair, yellow being dominant over green His data, however, do not furnish the necessary proof for his hypothesis. The males are uniformly of the same color, and he was thus able to assign to the male used in a given mating the particular genetic constitution necessary to explain the proportions obtained in the progeny; but apparently the tests necessary to determine the correctness of his assumptions were not made.

The fact, as shown by Pantel and de Sinety (1918), that coloration in several other species of walking-sticks in addition to Dixippus, is dependent upon the environment, suggests the desirability of reexamining the behavior in Clitumnus from this viewpoint.

Among a total of several thousand individuals reared in our cultures, two males and one gymandromorph appeared. The sporadic occurence of males and gymandromorphs has also been reported by others. Nachtsheim (1922) has suggested that non-disjunction is responsible for their appearance. In the Orthoptera generally, the female is characterized by the presence of 
two X-chromosomes, the male by one. The loss of one X-chromosome by non-disjunction or in any other manner would presumably recult in maleness. If the loss occurred before the first somatic division, the entire individual would be male,--if it occurred later a gynandromorph would presumably result. In the gynandromorph mentioned, the left side throughout the entire length was typically typically female, the right side was male, suggesting that the loss of an X-chromosome had occurred at the first somatic division.

One of the males was placed in a cage with several females which had just attained sexual maturity. Only females appeared in their progenies. Copulation was not observed, but the insects were seldom examined at night. Nachsheim mated one of the males which appeared in his cultures with females and observed repeated copulation but with no effect upon the sex of the progeny, - as in the progenies resulting from unmated individuals, all were females. Since, according to Pehani (1924) normal spermatozoa were produced by one of the sporadic males studied by him, it appears that the eggs have lost their capacity for fertilization.

In forms whose eggs have the ability to develop parthenogenetically and where parthenogenetic reproduction results exclusively in females, a trend in the sex ratio is automatically set up, which must end in the ultimate elimination of males unless this effect is offset by some factor or factors such as greater viability of males or of male-producing spermatozoa, which tend to distort the sex ratio in the opposite direction. In the Orthoptera, forms having the capacity for parthenogenetic reproduction are known in which males are never found, others in which males are rare, and still others in which the sex ratio is near equality. It must be concluded that forms in the last category have developed the capacity for parthenogenesis only recently or, as already suggested, that the trend toward an excess of females, which would otherwise be an inevitable concommitant of thelyotokous parthenogenesis, is being offset by other factors tending toward an excess of males. 


\section{Literature.}

Dobkiewicz, L. von. Einfluss der äusseren Umgebung auf die Farbung der indischen Stabheuschrecken, Dixippus morosus Biol. Zentralbl., 32. 1912, pp. 661-663.

Fryer, J. C. F. Preliminary Note on some Experiments with a Polymorphic Phasmid. Journ. Genetics, 3, 1913-1914, pp. 107-111.

Nachtsheim, H. Parthenogenese, Gynandromorphismus und Geschlechtsbestimmung bei Phasmiden. Zeitschr. f. Abst. u. Vererbgsl., 30: 287-289. 1923.

Pantel, J. and de Sinety, R. (1918). Réaction chromatique et non chromatique de quelques phasmides (Orthopt.) Bulletin Biologique, 52: 177-283. 

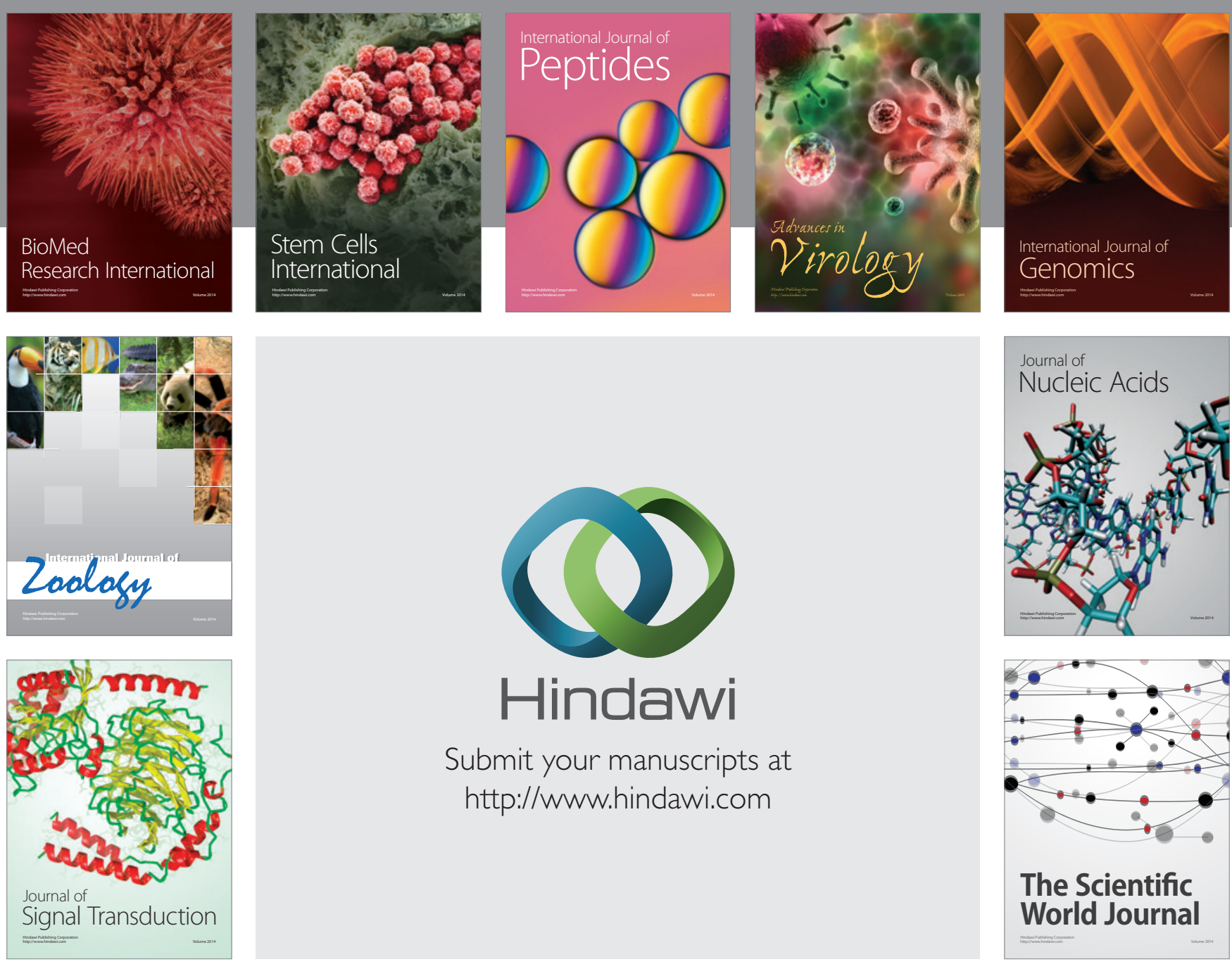

Submit your manuscripts at

http://www.hindawi.com
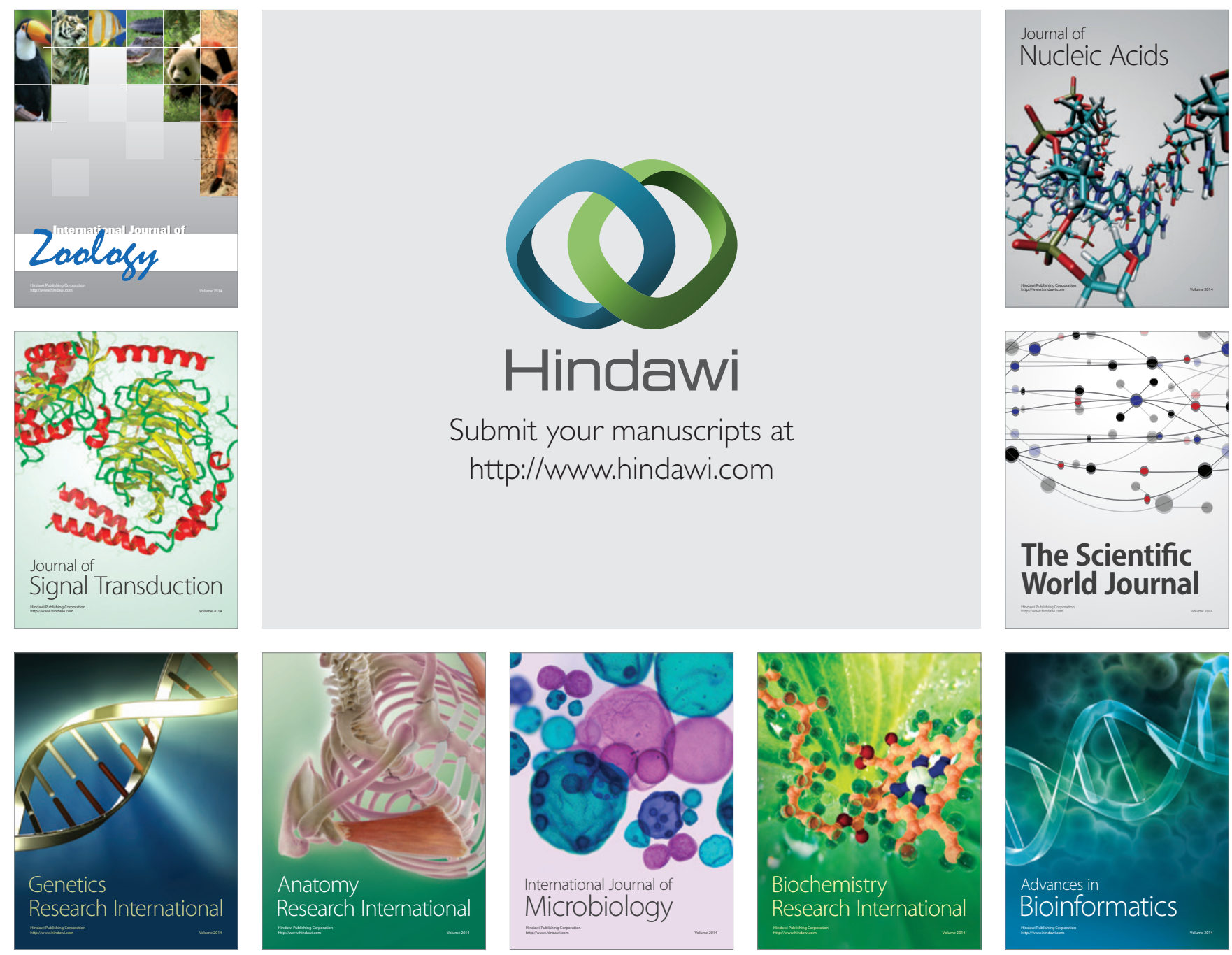

The Scientific World Journal
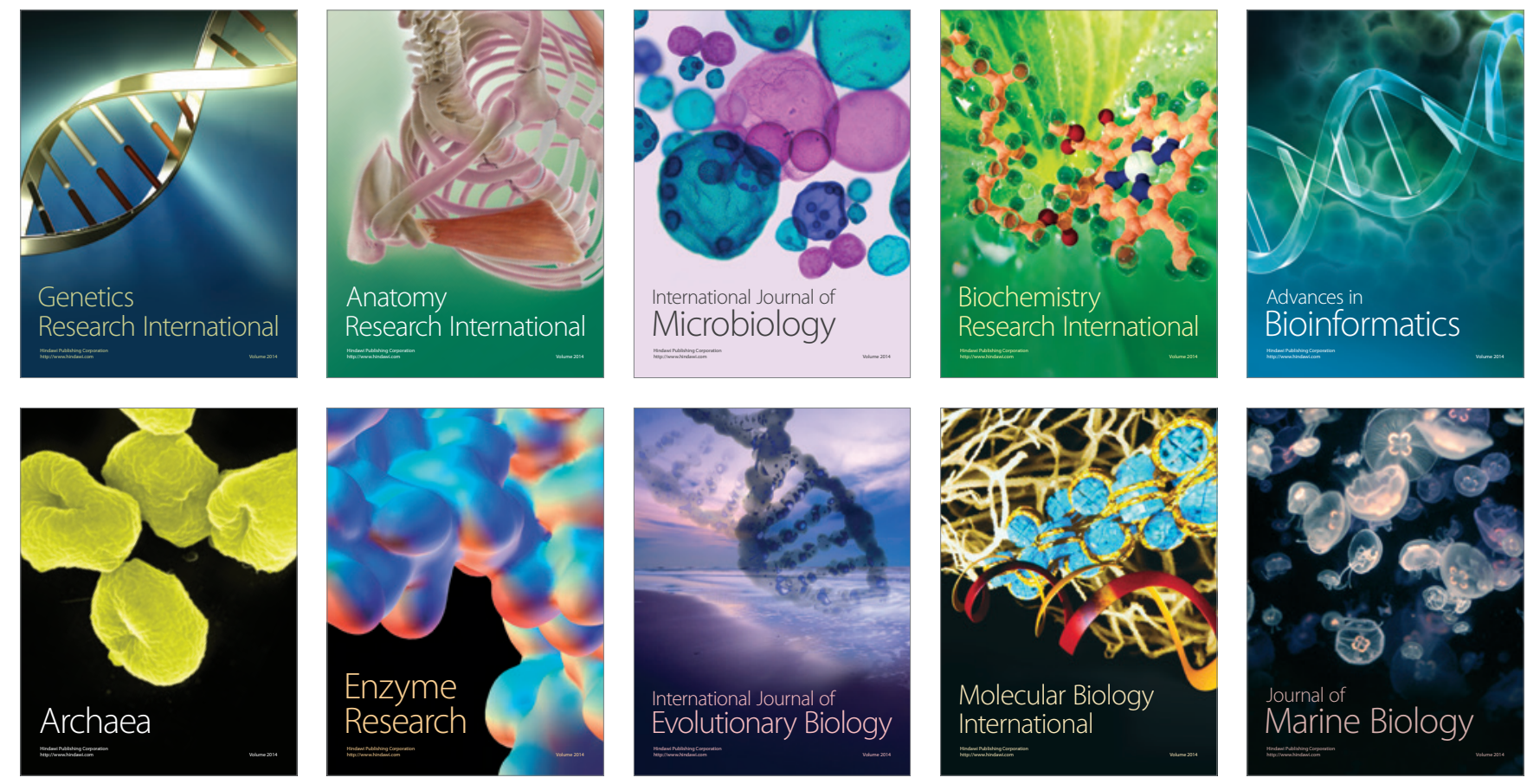